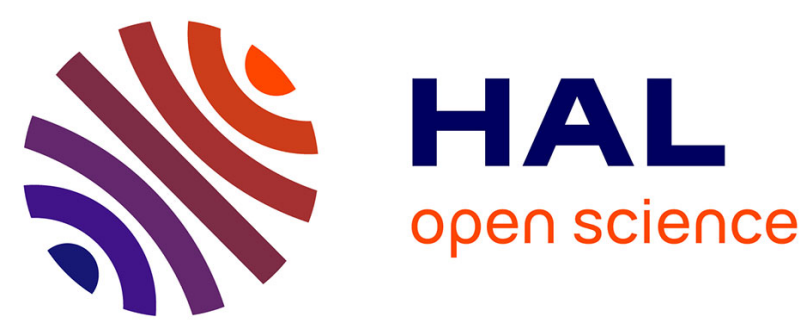

\title{
Singular mass matrix and redundant constraints in unilaterally constrained Lagrangian and Hamiltonian systems
}

\author{
Bernard Brogliato, Daniel Goeleven
}

\section{- To cite this version:}

Bernard Brogliato, Daniel Goeleven. Singular mass matrix and redundant constraints in unilaterally constrained Lagrangian and Hamiltonian systems. Multibody System Dynamics, 2015, 35 (1), pp.3961. 10.1007/s11044-014-9437-4. hal-01088286

\section{HAL Id: hal-01088286 \\ https://hal.inria.fr/hal-01088286}

Submitted on 29 Oct 2017

HAL is a multi-disciplinary open access archive for the deposit and dissemination of scientific research documents, whether they are published or not. The documents may come from teaching and research institutions in France or abroad, or from public or private research centers.
L'archive ouverte pluridisciplinaire HAL, est destinée au dépôt et à la diffusion de documents scientifiques de niveau recherche, publiés ou non, émanant des établissements d'enseignement et de recherche français ou étrangers, des laboratoires publics ou privés. 


\title{
Singular mass matrix and redundant constraints in unilaterally constrained Lagrangian and Hamiltonian systems
}

\author{
Bernard Brogliato • Daniel Goeleven
}

\begin{abstract}
This article deals with the analysis of the contact complementarity problem for Lagrangian systems subjected to unilateral constraints, and with a singular mass matrix and redundant constraints. Previous results by the authors on existence and uniqueness of solutions of some classes of variational inequalities are used to characterize the well-posedness of the contact problem. Criteria involving conditions on the tangent cone and the constraints gradient are given. It is shown that the proposed criteria easily extend to the case where the system is also subjected to a set of bilateral holonomic constraints, in addition to the unilateral ones. In the second part, it is shown how basic convex analysis may be used to show the equivalence between the Lagrangian and the Hamiltonian formalisms when the mass matrix is singular.
\end{abstract}

Keywords Singular Lagrangian system · Singular Hamiltonian system · Unilateral constraint · Bilateral holonomic constraint · Complementarity conditions · Mixed linear complementarity problem $\cdot$ Recession cone $\cdot$ Convex analysis $\cdot$ Redundant constraints . Legendre-Fenchel transformation

\section{Introduction}

Let us consider a Lagrangian system with Lagrangian function $L(q, \dot{q})=\frac{1}{2} \dot{q}^{\mathrm{T}} M(q) \dot{q}-$ $V(q), q \in \mathbb{R}^{n}$ the generalized coordinate vector, $\dot{q}$ the generalized velocity vector ( $q$ belongs to the configuration space $\mathcal{C}$ and $\dot{q}$ to the tangent space $T_{q} \mathcal{C}$. However, since we do not adopt any differential geometry point of view in this paper, we shall consider all quantities

\footnotetext{
B. Brogliato

INRIA, Bipop team-project, ZIRST Montbonnot, 655 avenue de l'Europe, 38334 Saint-Ismier cedex, France

e-mail: bernard.brogliato@inria.fr

D. Goeleven

Laboratoire PIMENT, Université de la Réunion, 97400 Saint-Denis, France

e-mail: goeleven@hotmail.fr
} 
as vectors with real components). The Lagrange dynamics is written when constraints are present as

$$
M(q) \ddot{q}+F(q, \dot{q}, t)=\nabla h(q) \lambda
$$

where $M(q)=M(q)^{\mathrm{T}}$ is the mass matrix, $F: \mathbb{R}^{n} \times \mathbb{R}^{n} \times \mathbb{R}_{+} \mapsto \mathbb{R}^{n}$ contains centripetal, Coriolis forces, forces that derive from a potential, and possible external forces like control inputs or perturbations, $\ddot{q}$ is the vector of generalized accelerations. The system is free, or subjected to constraints (bilateral $h(q)=0$, or unilateral $h(q) \geq 0$ ) represented by a differentiable function $h: \mathbb{R}^{n} \mapsto \mathbb{R}^{m}$ with gradient $=\nabla h(q)$, and $\lambda \in \mathbb{R}^{m}$ is the vector of Lagrange multipliers associated with the constraints. The mass matrix $M(q) \in \mathbb{R}^{n \times n}$ is often supposed to be positive definite. If in addition the constraints are functionally independent, then both the acceleration and the multipliers can be calculated uniquely. In the case of bilateral constraints, this boils down to solving a linear equation obtained with the Karush-Kuhn-Tucker matrix, stemming from the so-called index-1 DAE formulation [21, 27, 39]:

$$
\left(\begin{array}{cc}
M(q) & -\nabla h(q) \\
\nabla h(q)^{\mathrm{T}} & 0
\end{array}\right)\left(\begin{array}{l}
\ddot{q} \\
\lambda
\end{array}\right)=\left(\begin{array}{c}
-F(q, \dot{q}, t) \\
-\left(\frac{d}{d t} \nabla h(q)^{\mathrm{T}}\right) \dot{q}
\end{array}\right) .
$$

If both $M(q)$ and $\nabla h(q)$ are full-rank matrices, one calculates $\lambda$ using the Schur complement of the KKT matrix in (2): $\lambda=A(q)^{-1} H_{0}(q, \dot{q}, t)$, where

$$
H_{0}(q, \dot{q}, t) \triangleq \nabla h(q)^{\mathrm{T}} M(q)^{-1} F(q, \dot{q}, t)-\frac{d}{d t}\left(\nabla h(q)^{\mathrm{T}}\right) \dot{q}
$$

and $A(q)=\nabla h(q)^{\mathrm{T}} M(q)^{-1} \nabla h(q) \in \mathbb{R}^{m \times m}$. When $M(q)$ and/or $\nabla h(q)$ become singular, uniqueness of $\ddot{q}$ and of $\nabla h(q) \lambda$ is guaranteed for any $F(q, \dot{q}, t)$ if and only if [21]:

$$
\operatorname{ker} M(q) \cap \operatorname{ker} \nabla h(q)^{\mathrm{T}}=\{0\},
$$

that is equivalent to $\operatorname{rank}[M(q) \nabla h(q)]=n$ (the result is stated assuming that the system lives on the constraint submanifold $\left\{(q, \dot{q}): h(q)=0, \nabla h(q)^{\mathrm{T}} \dot{q}=0\right\}$, so that the second equality in (2) is satisfied). Uniqueness of $\lambda$ requires that $\nabla h(q)$ be full rank. The wellposedness of (2), together with the initial position and velocity compatibility with the constraint manifold, guarantees the existence and uniqueness of solutions to the constrained Lagrangian dynamics. Numerical methods also have to solve discretized versions of (2).

In the case of unilateral constraints, inducing complementarity conditions $0 \leq \lambda \perp$ $h(q) \geq 0$, and under the assumption that $M(q)$ has rank $n$, one computes the so-called contact linear complementarity problem $(L C P)[15,28]$ :

$$
0 \leq \lambda \perp A(q) \lambda+H_{0}(q, \dot{q}, t) \geq 0
$$

where $A(q)$ is called the Delassus' matrix in the literature on unilaterally constrained systems. The process followed to obtain the contact LCP may be seen as an extension of the index reduction of the bilateral DAE case. The conditions for the solvability, the feasibility and the uniqueness of solutions of the LCP in (4) are given in [15, Propositions 1, 2, 3], building on previous results by Moreau [30, 31] and Lötstedt [28]. It is seen from the results in $[21, \S 1,2]$ and $[15$, Propositions 1, 2, 3] that despite the linear equation in (2) and the contact LCP in (4) involving the same terms (in particular the Delassus's matrix is present in both), their well-posedness conditions differ. For instance, solvability of (2) relies on the above rank condition from a basic result in linear algebra (see, e.g. [10, Proposition 6.1.7]), while the solvability of (4) relies on complementarity theory [15, Proposition 3]. 
The above results on the contact LCP all rely on the assumption that $M(q)$ is positive definite. However, it is known that the rank of $M(q)$ may drop to $r<n$, hence it becomes positive semi-definite only $[3,4,8,12,23,25,38-41]$. The analysis of bilaterally constrained systems when the mass matrix has low rank has been made in [20-22, 27, 37, 42, 43]. The analysis of mixed bilaterally/unilaterally constrained systems is made in [15]. In this case one obtains a contact LCP with an augmented Delassus' matrix $A_{c}(q)$, which is distorted by the bilateral constraints effects. It is shown that even if $M(q)$ has full rank, the contact LCP may be ill-posed, i.e. $A_{c}(q)$ may be positive semi-definite only. Criteria are provided in [15] which guarantee that $A_{c}(q)$ is positive definite.

The case when unilateral constraints act on the system, and when $r<n$, however, has received no attention, to the best of the authors' knowledge. This is the topic of this article, which is dedicated to the analysis of the contact complementarity problem when low rank conditions on both $M(q)$ and $\nabla h(q)$ are present. The article is organized as follows: Some tools from convex analysis are recalled in the next paragraph. The contact complementarity problem, which takes the form of a mixed linear complementarity problem (MLCP), is constructed in Sect. 2. Its well-posedness is analyzed in Sect. 3. We show in Sect. 4 that the presence of holonomic bilateral constraints, in addition to the unilateral ones, may be treated within the foregoing framework. Section 5 shows how the MLCP may be transformed into an LCP, and paves the way towards Sect. 6 which is dedicated to proving the equivalence between degenerated Lagrangian and Hamiltonian systems with unilateral constraints. Two simple examples are treated in Sect. 7. Conclusions end the article in Sect. 8, and some mathematical results are given in Appendices A and B.

Notation and definitions. Let $h: \mathbb{R}^{n} \mapsto \mathbb{R}^{m}$ be differentiable. Its gradient at $q$ is $\nabla h(q) \in$ $\mathbb{R}^{n \times m}$, and its Jacobian is $\frac{\partial h}{\partial q}(q)=\nabla h(q)^{\mathrm{T}} \in \mathbb{R}^{m \times n}$, where the superscript $T$ means transpose. Given a vector $x \in \mathbb{R}^{n}, x \geq 0$ means $x_{i} \geq 0$ for all its entries $x_{i}$ (same for $>0$ ). Given a matrix $A \in \mathbb{R}^{n \times m}, \operatorname{ker}(A)$ is its kernel and $\operatorname{Im}(A)$ is its range, or image. Given two vectors $x$ and $y$, the product $\langle x, y\rangle=x^{\mathrm{T}} y$.

Mathematical tools in convex analysis. The next mathematical tools are mainly taken from classical references $[11,19,24,35]$. Let $f: \mathbb{R}^{n} \rightarrow \mathbb{R} \cup\{+\infty\}$ be a proper convex and lower semi-continuous function, we denote by $\operatorname{dom}(f):=\left\{x \in \mathbb{R}^{n}: f(x)<+\infty\right\}$ the domain of the function $f(\cdot)$. The epigraph of $f(\cdot)$ is the set epi $(f):=\left\{(x, \alpha) \in \mathbb{R}^{n} \times \mathbb{R}: \alpha \geq f(x)\right\}$.

Recall that the Fenchel transform $f^{\star}(\cdot)$ of $f(\cdot)$ is the proper, convex and lower semicontinuous function defined by

$$
\left(\forall z \in \mathbb{R}^{n}\right): \quad f^{\star}(z)=\sup _{x \in \operatorname{dom}(f)}\{\langle x, z\rangle-f(x)\} .
$$

The subdifferential $\partial f(x)$ of $f(\cdot)$ at $x \in \mathbb{R}^{n}$ is defined by

$$
\partial f(x)=\left\{\omega \in \mathbb{R}^{n}: f(v)-f(x) \geq\langle\omega, v-x\rangle, \forall v \in \mathbb{R}^{n}\right\} .
$$

We denote by $\operatorname{Dom}(\partial f):=\left\{x \in \mathbb{R}^{n}: \partial f(x) \neq \emptyset\right\}$ the domain of the subdifferential operator $\partial f: \mathbb{R}^{n} \rightarrow \mathbb{R}^{n}$. Recall that (see, e.g. [7, Theorem 2, Chap. 10, Sect. 3]):

$$
\operatorname{Dom}(\partial f) \subset \operatorname{dom}(f) \subset \overline{\operatorname{Dom}(\partial f)} \text {. }
$$

Let $x_{0}$ be any element in the domain $\operatorname{dom}(f)$ of $f(\cdot)$, the recession function $f_{\infty}(\cdot)$ of $f(\cdot)$ is defined by

$$
\left(\forall x \in \mathbb{R}^{n}\right): \quad f_{\infty}(x)=\lim _{\lambda \rightarrow+\infty} \frac{1}{\lambda} f\left(x_{0}+\lambda x\right) .
$$



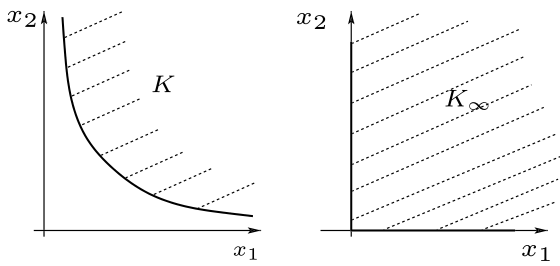

(a) Example 3 .
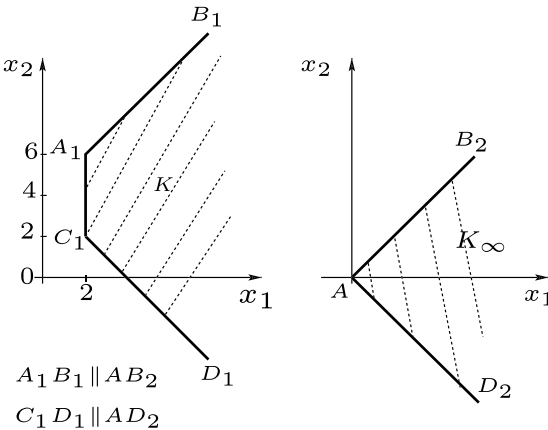

(b) Example 4 .

Fig. 1 Recession cones

The function $f_{\infty}: \mathbb{R}^{n} \rightarrow \mathbb{R} \cup\{+\infty\}$ is a proper convex and lower semi-continuous function which describes the asymptotic behavior of $f(\cdot)$.

Let $K \subset \mathbb{R}^{n}$ be a nonempty closed convex set. Let $x_{0}$ be any element in $K$. The recession cone of $K$ is defined by

$$
K_{\infty}=\bigcap_{\lambda>0} \frac{1}{\lambda}\left(K-x_{0}\right)=\left\{u \in \mathbb{R}^{n}: x+\lambda u \in K, \forall \lambda \geq 0, \forall x \in K\right\} .
$$

The set $K_{\infty}$ is a nonempty closed convex cone that is described in terms of the directions which recede from $K$. The indicator function of $K$ is $\Psi_{K}(x)=0$ if $x \in K, \Psi_{K}(x)=+\infty$ if $x \notin K$.

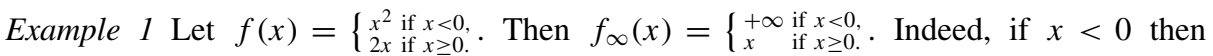
$\lim _{\lambda \rightarrow+\infty} \frac{1}{\lambda} f(\lambda x)=\lim _{\lambda \rightarrow+\infty} \lambda x^{2}=+\infty$ while if $x \geq 0$ then $\lim _{\lambda \rightarrow+\infty} \frac{1}{\lambda} f(\lambda x)=$ $\lim _{\lambda \rightarrow+\infty} x=x$.

Example 2 Let $M \in \mathbb{R}^{n \times n}$ be a positive semi-definite and symmetric matrix, $q \in \mathbb{R}^{n}$ and $f(x)=\frac{1}{2} x^{\mathrm{T}} M x+q^{\mathrm{T}} x$. Then $f_{\infty}(x)=\left\{\begin{array}{c}q^{\mathrm{T}} x \text { if } x \in \operatorname{ker}(M), \\ +\infty \text { if } x \notin \operatorname{ker}(M) .\end{array}\right.$.

Example 3 Let $K=\left\{\left(x_{1}, x_{2}\right) \in \mathbb{R}^{2}: x_{1}>0, x_{1} x_{2} \geq 1\right\}$. Then $K_{\infty}=\left\{\left(x_{1}, x_{2}\right) \in \mathbb{R}^{2}: x_{1} \geq\right.$ $\left.0, x_{2} \geq 0\right\}$. This is depicted in Fig. 1a.

Example 4 Let $K=\left\{\left(x_{1}, x_{2}\right) \in \mathbb{R}^{2}: x_{1} \geq 2,-x_{1}+4 \leq x_{2} \leq x_{1}+4\right\}$. Then $K_{\infty}=\left\{\left(x_{1}, x_{2}\right) \in\right.$ $\left.\mathbb{R}^{2}: x_{1} \geq 0,-x_{1} \leq x_{2} \leq x_{1}\right\}$. This is depicted in Fig. $1 \mathrm{~b}$.

Let us here recall some important properties of the recession function and recession cone (see, e.g. [34, Chaps. 8 and 9], or [11, Proposition 1.4.8]):

\section{Proposition 1}

(a) Let $f_{1}: \mathbb{R}^{n} \rightarrow \mathbb{R} \cup\{+\infty\}$ and $f_{2}: \mathbb{R}^{n} \rightarrow \mathbb{R} \cup\{+\infty\}$ be two proper, convex and lower semi-continuous functions. Suppose that $f_{1}+f_{2}$ is proper. Then

$$
\left(\forall x \in \mathbb{R}^{n}\right): \quad\left(f_{1}+f_{2}\right)_{\infty}(x)=\left(f_{1}\right)_{\infty}(x)+\left(f_{2}\right)_{\infty}(x) .
$$




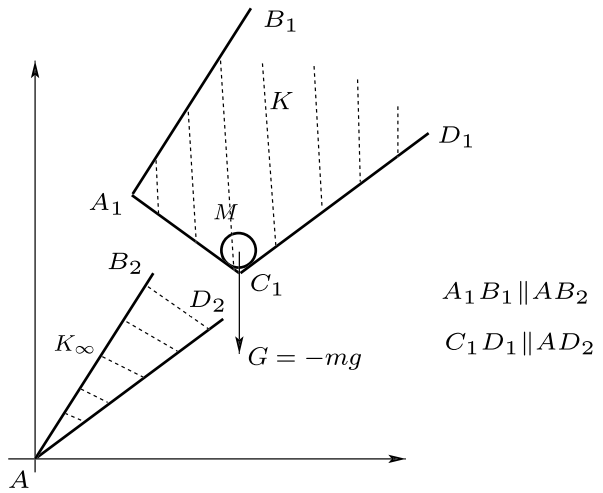

(a) First case of example 6 .

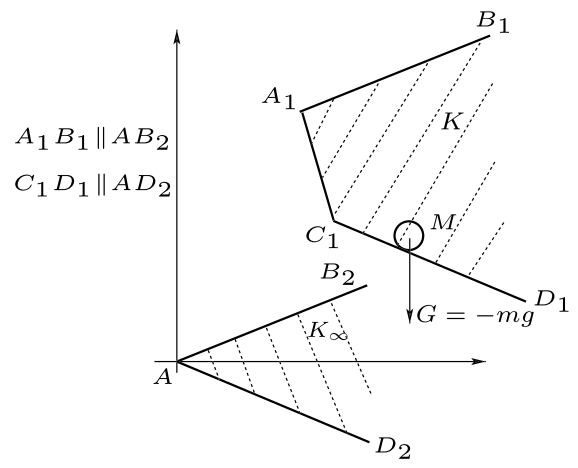

(b) Second case of example 6 .

Fig. 2 Equilibrium positions

(b) Let $f: \mathbb{R}^{n} \rightarrow \mathbb{R} \cup\{+\infty\}$ be a proper, convex and lower semi-continuous function and let $K$ be a nonempty closed convex set such that $f+\Psi_{K}$ is proper (equivalently, $\operatorname{dom}(f) \cap$ $K$ is nonempty). Then

$$
\left(\forall x \in \mathbb{R}^{n}\right): \quad\left(f+\Psi_{K}\right)_{\infty}(x)=f_{\infty}(x)+\left(\Psi_{K}\right)_{\infty}(x) .
$$

(c) Let $K \subset \mathbb{R}^{n}$ be a nonempty, closed and convex set. Then

$$
\begin{gathered}
\left(\forall x \in \mathbb{R}^{n}\right): \quad\left(\Psi_{K}\right)_{\infty}(x)=\Psi_{K_{\infty}}(x), \\
\left(\forall x \in K, e \in K_{\infty}\right): \quad x+e \in K .
\end{gathered}
$$

(d) If $K \subset \mathbb{R}^{n}$ is a nonempty closed and convex cone, then $K_{\infty}=K$.

Example 5 Let $K=[a, b],(a, b \in \mathbb{R}, a<b)$. The set $K$ is bounded and thus $K_{\infty}=\{0\}$. It results that

$$
\left(\Psi_{K}\right)_{\infty}(x)=\Psi_{\{0\}}= \begin{cases}+\infty & \text { if } x \neq 0 \\ 0 & \text { if } x=0 .\end{cases}
$$

Example 6 Let us consider a ball $M$ subjected to its weight $G$ and constrained to stay in a box $K$. In case of Fig. 2a, all directions of $K_{\infty}$ make an obtuse angle with the direction of the applied force, i.e. $\left(\forall x \in K_{\infty}\right): G^{\mathrm{T}} x<0$. In that case, we note that the ball admits a unique equilibrium position. The condition $\left(\forall x \in K_{\infty}\right): G^{\mathrm{T}} x<0$ may also be written as

$$
\left(\forall x \in \mathbb{R}^{2}\right): \quad G^{\mathrm{T}} x+\Psi_{K_{\infty}}(x)<0 .
$$

Let us now set $U(x)=G^{\mathrm{T}} x+\Psi_{K}(x)$. The mapping $x \mapsto G^{\mathrm{T}} x$ is the potential energy corresponding to the gravity force while $\Psi_{K}(x)$ is a penalty energy associated with the constraints. Using (8), we see that $U_{\infty}(x)=G^{\mathrm{T}} x+\Psi_{K_{\infty}}(x)$, and thus condition (11) is equivalent to $\left(\forall x \in \mathbb{R}^{2}\right): U_{\infty}(x)<0$. In case of Fig. $2 \mathrm{~b}$, there exists a direction $v_{\infty} \in K_{\infty}$ making an acute angle with the direction of the applied force, i.e. $G^{\mathrm{T}} v_{\infty}>0$, and we see clearly that there is no possible equilibrium position for the ball. This simple example shows the importance of the tools of recession to determine equilibrium conditions in the case of systems subjected to unilateral constraints. 
Example 7 A polyhedral set is defined as the set of solutions to a finite set of linear inequalities, i.e.

$$
P(A, b)=\left\{x \in \mathbb{R}^{n}: A x \geq b\right\},
$$

where $A \in \mathbb{R}^{m \times n}$ and $b \in \mathbb{R}^{m}$. It is clear that $P(A, b)$ is a closed convex set. A function $f: \mathbb{R}^{n} \rightarrow \mathbb{R} \cup\{+\infty\}$ whose epigraph is polyhedral is called a polyhedral function [35, p. 172]. Suppose that $P(A, b) \neq \emptyset$. Then there exists $x_{0} \in \mathbb{R}^{n}: A x_{0} \geq b$ and

$$
P(A, b)_{\infty}=\bigcap_{\lambda>0} \frac{1}{\lambda}\left(P(A, b)-x_{0}\right)=P(A, 0) .
$$

Indeed,

$$
\begin{aligned}
x \in P(A, b)_{\infty} & \Leftrightarrow \quad(\forall \lambda>0): \quad A\left(\lambda x+x_{0}\right) \geq b \\
& \Leftrightarrow \quad(\forall \lambda>0): \quad \lambda A x+A x_{0}-b \geq 0 \quad \Leftrightarrow \quad A x \geq 0,
\end{aligned}
$$

where one checks that (10) is satisfied.

Let $K$ be a subset of $\mathbb{R}^{n}$. The polar cone of $K$ is defined by

$$
K^{+}=\left\{w \in \mathbb{R}^{n}:\langle w, v\rangle \leq 0, \forall v \in K\right\} .
$$

The dual cone of $K$ is defined by the relation

$$
K^{*}=-K^{+}
$$

Let $K$ be a nonempty subset of $\mathbb{R}^{n}$. To every point $x \in K$, we can associate the set

$$
T_{K}(x)=\left\{y \in \mathbb{R}^{n}: \exists\left\{x_{n}\right\} \subset K,\left\{\lambda_{n}\right\} \subset \mathbb{R}_{+}, \lambda_{n} \searrow 0, x_{n} \rightarrow x, \frac{x_{n}-x}{\lambda_{n}} \rightarrow y\right\}
$$

called the tangent cone of $K$ at $x$. The normal cone to $K$ at $x$ is defined as the polar cone of $T_{K}(x)$, i.e.

$$
N_{K}(x)=T_{K}(x)^{+}
$$

If in addition $K$ is convex then

$$
N_{K}(x)=\left\{z \in \mathbb{R}^{n}:\langle z, v-x\rangle \leq 0 \text { for all } v \in K\right\}
$$

and $N_{K}(x)^{+}=T_{K}(x)$. It represents the cone generated by the normals to the set $K$ at $x$, and is an outwards cone. If $K$ is a nonempty closed convex set, then one has $\partial \Psi_{K}(\cdot)=N_{K}(\cdot)$ and both the tangent and normal cones are convex closed sets.

\section{The contact complementarity problem}

Let us consider (1) subjected to a set of $m$ unilateral constraints $h(q) \geq 0$, giving rise to complementarity conditions $0 \leq \lambda \perp h(q) \geq 0$. During persistent contact phases with the $m$ constraint surfaces, the complementarity can be written at the acceleration level as $0 \leq$ $\lambda(t) \perp \frac{d^{2}}{d t^{2}} h(q(t)) \geq 0$ (if not all $m$ constraints are active, then one constructs the LCP taking 
into account only the active constraints). We further assume that the acceleration is rightcontinuous, i.e. $\ddot{q}(t)=\ddot{q}\left(t^{+}\right)$. The same holds for the multiplier $\lambda(\cdot)$. Developing a bit more, one obtains

$$
0 \leq \lambda(t) \perp \nabla h(q)^{\mathrm{T}} \ddot{q}(t)+\frac{d}{d t}\left(\nabla h(q(t))^{\mathrm{T}}\right) \dot{q}(t) \geq 0 .
$$

Let us denote $x \triangleq \ddot{q}(t), \lambda \triangleq \lambda(t)$ and $H(q, \dot{q}) \triangleq \frac{d}{d t}\left(\nabla h(q(t))^{\mathrm{T}}\right) \dot{q}(t)$. Then (1) and (15) give rise to

$$
\left\{\begin{array}{l}
M(q) x+F(q, \dot{q}, t)-\nabla h(q) \lambda=0, \\
0 \leq \lambda \perp \nabla h(q)^{\mathrm{T}} x+H(q, \dot{q}) \geq 0 .
\end{array}\right.
$$

The set of equations in (16) is a mixed LCP (MLCP) [1, Definition 12.22], which is a combination between an LCP and linear equations. If $M(q)$ has rank $n$ it reduces to the LCP in (4). This MLCP is the counterpart of (2) for unilaterally constrained systems. In order to analyze the well-posedness of this MLCP, let us transform it into a suitable form. First, it follows from a basic convex analysis result that

$$
0 \leq \lambda \perp \nabla h(q)^{\mathrm{T}} x+H(q, \dot{q}) \geq 0 \quad \Leftrightarrow \quad \lambda \in-\partial \Psi_{K}\left(\nabla h(q)^{\mathrm{T}} x+H(q, \dot{q})\right)
$$

with $K=\mathbb{R}_{+}^{m}$. Thus the MLCP is rewritten as the inclusion

$$
M(q) x+F(q, \dot{q}, t) \in-\nabla h(q) \partial \Psi_{K}\left(\nabla h(q)^{\mathrm{T}} x+H(q, \dot{q})\right) .
$$

Here $q$ and $\dot{q}$ are considered as parameters, the argument of the subdifferential being $x$. We set

$$
\tilde{K} \triangleq\left\{z \in \mathbb{R}^{m}: z+H(q, \dot{q}) \in K\right\}=K-H(q, \dot{q}) .
$$

Let us make the following:

Assumption 1 We suppose that

$$
\operatorname{Im}\left(\nabla h(q)^{\mathrm{T}}\right) \cap \tilde{K} \neq \emptyset .
$$

Let us also set

$$
\Phi=\left\{x \in \mathbb{R}^{n}: \nabla h(q)^{\mathrm{T}} x+H(q, \dot{q}) \in K\right\} .
$$

Assumption 1 entails that $\Phi$ is nonempty. It is also clear that $\Phi$ is closed and convex. This set (as well as $\tilde{K}$ ) should be denoted with arguments as $\Phi(q, \dot{q})$; however, to lighten the notation, we drop the arguments. We have

$$
\Psi_{\Phi}(x)=\Psi_{K}\left(\nabla h(q)^{\mathrm{T}} x+H(q, \dot{q})\right)=\Psi_{\tilde{K}}\left(\nabla h(q)^{\mathrm{T}} x\right) .
$$

Let us first consider $\Psi_{\Phi}(\cdot)$ as the composition mapping

$$
\Psi_{\Phi}(x)=\Psi_{\tilde{K}}\left(\nabla h(q)^{\mathrm{T}} x\right) .
$$

The set $\tilde{K}$ is a polyhedral set in $\mathbb{R}^{m}$ (see (12)). Indeed,

$$
\tilde{K}=P\left(I_{m \times m},-H(q, \dot{q})\right) .
$$


It results that the indicator function $\Psi_{\tilde{K}}(\cdot)$ is a polyhedral function. Then, by Theorem 1 in Appendix B and Assumption 1, it follows that (here $h(\cdot)=\Psi_{\tilde{K}}(\cdot), A=\nabla h(q)^{\mathrm{T}}$ and $\left.f(\cdot)=\Psi_{\Phi}(\cdot)\right)$

$$
\partial \Psi_{\Phi}(x)=\nabla h(q) \partial \Psi_{\tilde{K}}\left(\nabla h(q)^{\mathrm{T}} x\right)
$$

We have also

$$
\partial \Psi_{\tilde{K}}\left(\nabla h(q)^{\mathrm{T}} x\right)=\partial \Psi_{K-H(q, \dot{q})}\left(\nabla h(q)^{\mathrm{T}} x\right)=\partial \Psi_{K}\left(\nabla h(q)^{\mathrm{T}} x+H(q, \dot{q})\right),
$$

and thus

$$
\nabla h(q) \partial \Psi_{K}\left(\nabla h(q)^{\mathrm{T}} x+H(q, \dot{q})\right)=\partial \Psi_{\Phi}(x) .
$$

Let us also notice that a necessary condition for the MLCP in (16) to possess at least one solution is that $\Phi$ is nonempty. This is guaranteed by Assumption $1 .^{1}$ Thus we have shown that the MLCP in (16) is equivalent, under the constraint qualification of Assumption 1, to the generalized equation

$$
\begin{aligned}
& M(q) x+F(q, \dot{q}, t) \in-\partial \Psi_{\Phi}(x) \\
& \quad \Leftrightarrow \quad \text { Find } x \in \Phi:\langle M(q) x+F(q, \dot{q}, t), v-x\rangle \geq 0 \text { for all } v \in \Phi,
\end{aligned}
$$

where the second formalism is a variational inequality.

Remark 1 Usually the contact problem is studied in terms of the multiplier from the LCP in (4). Here we address the problem by studying the existence and uniqueness of the acceleration $x$ in (21).

\section{Well-posedness of the MLCP}

If $\operatorname{rank}(M(q))=n$ then Theorem 6 in [2] applies to show the existence and uniqueness of $x$ (hence providing an alternative proof of the result shown by Moreau in [31]). By elementary convex analysis, one finds that the generalized equation (21) has a unique solution given by an orthogonal projection in the kinetic metric (see [15, §4.4] for further developments):

$$
x=\operatorname{proj}_{M(q)}\left[\Phi ;-M(q)^{-1} F(q, \dot{q}, t)\right] .
$$

However, the case of interest here is when $r<n$. Using results from [2], we are going to characterize the existence (solvability) and uniqueness of solutions to (21). Let us first recall the following result, where we concatenate Theorem 3 and Corollary 4 in [2] (Corollary 5 in [2] cannot be applied unless $H(q, \dot{q})=0$ in which case $\Phi$ is a closed convex cone). They concern variational inequalities of the form: Find $u \in \mathbb{R}^{n}$ such that

$$
\langle\mathbf{M} u+\mathbf{q}, v-u\rangle+\varphi(v)-\varphi(u) \geq 0, \quad \forall v \in \mathbb{R}^{n}
$$

where $\mathbf{M} \in \mathbb{R}^{n \times n}$ is a real matrix, $\mathbf{q} \in \mathbb{R}^{n}$ a vector and $\varphi: \mathbb{R}^{n} \rightarrow \mathbb{R} \cup\{+\infty\}$ a proper convex and lower semicontinuous function. The analogy with the generalized equation in (21) is

\footnotetext{
${ }^{1}$ Another constraint qualification guaranteeing nonemptyness of $\tilde{K}$, hence of $\Phi$, is range $\left(\nabla h(q)^{\mathrm{T}}\right)-$ $\mathbb{R}_{+}^{m}=\mathbb{R}^{m}$, which does not imply that $\nabla h(q)^{\mathrm{T}}$ has rank $m$ [17].
} 
clear taking $\varphi(\cdot)=\Psi_{\Phi}(\cdot)$, thus restricting the variation of $v$ to $\Phi, \mathbf{q}=F(q, \dot{q}, t)$ and $\mathbf{M}=$ $M(q)$.

The problem in (23) is denoted as $V I(\mathbf{M}, \mathbf{q}, \varphi)$ in the next proposition. We also set

$$
\mathcal{K}(\mathbf{M}, \varphi)=\left\{x \in \mathbb{R}^{n} \mid \mathbf{M} x \in\left(\operatorname{dom}\left(\varphi_{\infty}\right)\right)^{*}\right\} .
$$

Note that $\left(\operatorname{dom}\left(\varphi_{\infty}\right)\right)^{*}$ is the dual cone of the domain of the recession function $\varphi_{\infty}$ while $(\operatorname{dom}(\varphi))_{\infty}$ is the recession cone of $\operatorname{dom}(\varphi)$.

Proposition 2 [2] Let $\varphi: \mathbb{R}^{n} \rightarrow \mathbb{R} \cup\{+\infty\}$ be a proper, convex and lower semicontinuous function with closed domain, and suppose that $\mathbf{M} \in \mathbb{R}^{n \times n}$ is positive semi-definite and symmetric.

(a) If $(\operatorname{dom}(\varphi))_{\infty} \cap \operatorname{ker}\{\mathbf{M}\} \cap \mathcal{K}(\mathbf{M}, \varphi)=\{0\}$ then for each $\mathbf{q} \in \mathbb{R}^{n}$, problem $\operatorname{VI}(\mathbf{M}, \mathbf{q}, \varphi)$ has at least one solution.

(b) Suppose that $(\operatorname{dom}(\varphi))_{\infty} \cap \operatorname{ker}\{\mathbf{M}\} \cap \mathcal{K}(\mathbf{M}, \varphi) \neq\{0\}$. If

$$
\langle\mathbf{q}, v\rangle+\varphi_{\infty}(v)>0, \quad \forall v \in \operatorname{dom}(\varphi)_{\infty} \cap \operatorname{ker}\{\mathbf{M}\} \cap \mathcal{K}(\mathbf{M}, \varphi), v \neq 0,
$$

then problem $\operatorname{VI}(\mathbf{M}, \mathbf{q}, \varphi)$ has at least one solution.

(c) If $u_{1}$ and $u_{2}$ denote two solutions of problem $V I(\mathbf{M}, \mathbf{q}, \varphi)$ then

$$
u_{1}-u_{2} \in \operatorname{ker}\{\mathbf{M}\}
$$

and

$$
\left\langle\mathbf{q}, u_{1}-u_{2}\right\rangle=\varphi\left(u_{2}\right)-\varphi\left(u_{1}\right) .
$$

(d) If

$$
\varphi(x+z)=\varphi(x), \quad \forall x \in \operatorname{dom}(\varphi), z \in \operatorname{ker}\{\mathbf{M}\}
$$

and

$$
\langle\mathbf{q}, e\rangle \neq 0, \quad \forall e \in \operatorname{ker}\{\mathbf{M}\}, e \neq 0,
$$

then problem $\operatorname{VI}(\mathbf{M}, \mathbf{q}, \varphi)$ has at most one solution.

Notice that the function $\varphi(\cdot)$ will never be strictly convex in our case (it is an indicator function) so that the strict convexity argument of Corollary 4 in [2] does not apply and is not recalled. Let us now apply Proposition 2 to (21). We need to perform some calculations on the recession and dual cones first to prepare the result statement.

We have

$$
\operatorname{dom}(\varphi)=\operatorname{dom}\left(\Psi_{\Phi}\right)=\Phi=P\left(\nabla h(q)^{\mathrm{T}},-H(q, \dot{q})\right) .
$$

Thus using (13),

$$
(\operatorname{dom}(\varphi))_{\infty}=\Phi_{\infty}=P\left(\nabla h(q)^{\mathrm{T}}, 0\right)=\left\{x \in \mathbb{R}^{n}: \nabla h(q)^{\mathrm{T}} x \geq 0\right\} .
$$

Let $S \triangleq\left\{q \in \mathbb{R}^{n}: h(q) \geq 0\right\}$ be the admissible domain of configurations. Suppose that all $m$ constraints are active, i.e. $h(q(t))=0$ for all $t \in I$ where $I$ is an interval (not reduced to a singleton), and suppose that the Mangasarian-Fromovitz constraint qualification (MFCQ) 
holds (see Appendix A). This constraint qualification reduces here to the existence of $v \in \mathbb{R}^{n}$ such that $\nabla h(q)^{\mathrm{T}} v>0$. Then we may write

$$
\left\{x \in \mathbb{R}^{n}: \nabla h(q)^{\mathrm{T}} x \geq 0\right\}=T_{S}(q) .
$$

From (9) one has $\varphi_{\infty}(\cdot)=\Psi_{\Phi_{\infty}}(\cdot)$. Thus the dual cone in (24) is given by

$$
\left(\operatorname{dom}\left(\varphi_{\infty}\right)\right)^{*}=\Phi_{\infty}^{*}
$$

Using the well known Farkas' lemma, we get

$$
\begin{aligned}
\Phi_{\infty}^{*} & =\left\{x \in \mathbb{R}^{n}: \exists \alpha \geq 0 \text { such that } x=\nabla h(q) \alpha\right\} \\
& =\left\{x \in \mathbb{R}^{n}: \exists \alpha_{1}, \ldots, \alpha_{m} \geq 0 \text { such that } x=\sum_{i=1}^{m} \nabla h_{i}(q) \alpha_{i}\right\} .
\end{aligned}
$$

Still assuming that all $m$ constraints are active and that the MFCQ holds, one obtains

$$
\Phi_{\infty}^{*}=\left(\operatorname{dom}\left(\varphi_{\infty}\right)\right)^{*}=-N_{S}(q)
$$

Consequently, using (24), we have

$$
\mathcal{K}(M, \varphi)=\left\{x \in \mathbb{R}^{n}: \exists \alpha \geq 0 \text { such that } M(q) x=\nabla h(q) \alpha\right\} .
$$

In the next propositions, $q(t), \dot{q}(t)$ and $t$ are considered as given parameters. Recall that by a solution of the MLCP one means a vector $x \in \mathbb{R}^{n}$ (and not a multiplier $\lambda$ ), since we are studying the variational inequality (21).

Remark 2 When the constraints are bilateral (i.e. $h(q)=0$ ), the tangent cone $T_{S}(q)$ and the normal $N_{S}(q)$ cone reduce to the usual tangent and normal subspaces to the submanifold $\Sigma=\left\{q \in \mathbb{R}^{n}: h(q)=0\right\}=S$, respectively. Then $T_{S}(q)=\operatorname{ker}\left(\nabla h(q)^{\mathrm{T}}\right)$ and $N_{S}(q)=\left(T_{S}(q)\right)^{\perp}=\operatorname{Im}(\nabla h(q))$.

Proposition 3 (MLCP solvability) Assume that the $m$ unilateral constraints are active, that Assumption 1 holds and that one of the following conditions is satisfied:

(i) $P\left(\nabla h(q)^{\mathrm{T}}, 0\right) \cap \operatorname{ker}(M(q))=\{0\}$,

(ii) $P\left(\nabla h(q)^{\mathrm{T}}, 0\right) \cap \operatorname{ker}(M(q)) \neq\{0\}$ and $F(q, \dot{q}, t)^{\mathrm{T}} v>0$ for all $v \in P\left(\nabla h(q)^{\mathrm{T}}, 0\right) \cap$ $\operatorname{ker}(M(q)), v \neq 0$.

Then the MLCP in (16) has at least one solution.

Proof It follows from Proposition 2(a) and (b). Notice that

$$
\begin{aligned}
\Phi_{\infty} & \cap \operatorname{ker}(M(q)) \cap \mathcal{K}\left(M(q), \Psi_{\Phi}\right) \\
= & \left\{x \in \mathbb{R}^{n}: \nabla h(q)^{\mathrm{T}} x \geq 0, M(q) x=0 \text { and } M(q) x=\nabla h(q) \alpha \text { for some } \alpha \geq 0\right\} \\
& =\left\{x \in \mathbb{R}^{n}: \nabla h(q)^{\mathrm{T}} x \geq 0 \text { and } M(q) x=0 \text { and } 0=\nabla h(q) \alpha \text { for some } \alpha \geq 0\right\} \\
& =\left\{x \in \mathbb{R}^{n}: \nabla h(q)^{\mathrm{T}} x \geq 0 \text { and } M(q) x=0\right\}=\Phi_{\infty} \cap \operatorname{ker}(M(q)) .
\end{aligned}
$$

The results follow using (28). 
Fig. 3 A system satisfying Proposition 4(ii); $S=\left\{q \in \mathbb{R}^{n}: h(q) \geq 0\right\}$

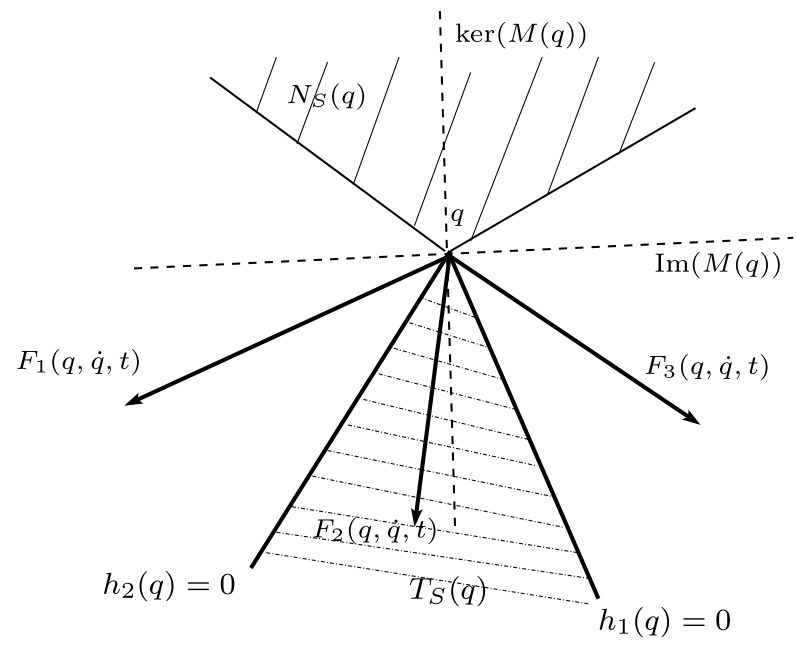

$S: \begin{array}{ll}h_{1}(q)>0 \\ & h_{2}(q)>0\end{array}$

If the MFCQ is satisfied and all $m$ constraints are active then using (29) we may rewrite the previous result as follows:

Proposition 4 (MLCP solvability) Suppose that all $m$ unilateral constraints are active, i.e. $h(q)=0$, and suppose that the MFCQ holds. Under Assumption 1 and if one of the following conditions is satisfied:

(i) $T_{S}(q) \cap \operatorname{ker}(M(q))=\{0\}$,

(ii) $T_{S}(q) \cap \operatorname{ker}(M(q)) \neq\{0\}$ and $F(q, \dot{q}, t)^{\mathrm{T}} v>0$ for all $v \in T_{S}(q) \cap \operatorname{ker}(M(q)), v \neq 0$,

then the MLCP in (16) has at least one solution.

Figure 3 depicts a case when Proposition 4(ii) is applicable, where the tangent and normal cones are the dashed areas. Exchanging $\operatorname{ker}(M(q))$ with $\operatorname{Im}(M(q))$ and vice versa, one obtains $T_{S}(q) \cap \operatorname{ker}(M(q))=\{0\}$.

Remark 3 (i) Condition (i) of Proposition 3 holds if $M(q)$ is invertible since in this case $\operatorname{ker}(M(q))=\{0\}$. (ii) If $M(q)$ is not invertible and $m=1$ then $P\left(\nabla h(q)^{\mathrm{T}}, 0\right) \cap$ $\operatorname{ker}(M(q)) \neq \emptyset$. Indeed, $\operatorname{dim}(\operatorname{ker}(M(q)))=v \geq 1$; let $\xi_{1}, \ldots, \xi_{v}$ be a basis of $\operatorname{ker}(M)$. Setting $\alpha_{i}=1$ when $\nabla h(q)^{\mathrm{T}} \xi_{i} \geq 0$ and $\alpha_{i}=-1$ when $\nabla h(q)^{\mathrm{T}} \xi_{i}<0$, we see that

$$
x=\alpha_{1} \xi_{1}+\cdots+\alpha_{\nu} \xi_{v} \in \operatorname{ker}(M(q))
$$

and

$$
\nabla h(q)^{\mathrm{T}} x=\alpha_{1} \nabla h(q)^{\mathrm{T}} \xi_{1}+\cdots+\alpha_{\nu} \nabla h(q)^{\mathrm{T}} \xi_{v} \geq 0
$$

Thus

$$
x \in P\left(\nabla h(q)^{\mathrm{T}}, 0\right) \cap \operatorname{ker}(M) .
$$


Fig. 4 A system satisfying Proposition 4(ii); $S=\left\{q \in \mathbb{R}^{n}: h(q) \geq 0\right\}$

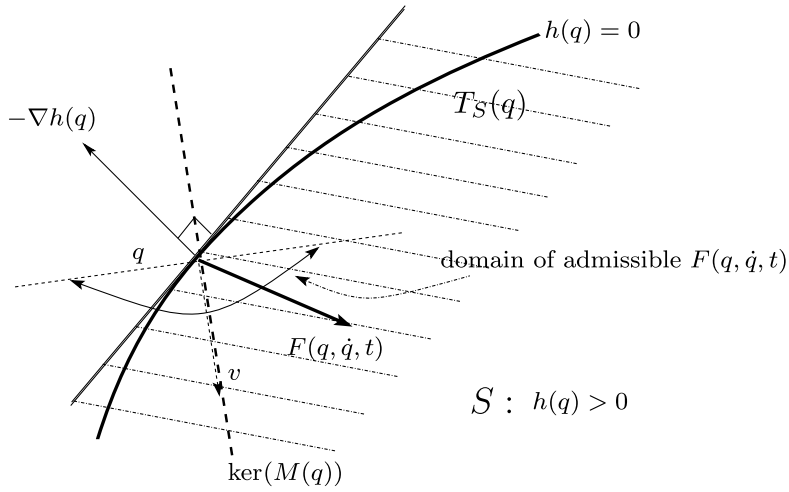

Thus if $m=1$ (there is only one active constraint at $q$ ), one has to resort to condition (ii) of Proposition 4. This is illustrated in Fig. 4. In this case the tangent cone is the dashed half space, while the normal cone is a half line generated by $-\nabla h(q)$.

Proposition 5 (MLCP uniqueness of solutions) (a) Let $\ddot{q}_{1}(t), \ddot{q}_{2}(t)$, and $\lambda_{1}(t), \lambda_{2}(t)$ be solutions of the MLCP (16). Then

$$
\left\{\begin{array}{l}
\ddot{q}_{1}(t)-\ddot{q}_{2}(t) \in \operatorname{ker}(M(q)), \\
\lambda_{1}(t)-\lambda_{2}(t) \in \operatorname{ker}(\nabla h(q)), \\
F(q, \dot{q}, t)^{\mathrm{T}}\left(\ddot{q}_{1}(t)-\ddot{q}_{2}(t)\right)=0 .
\end{array}\right.
$$

(b) Suppose that

$$
\begin{cases}\nabla h(q)^{\mathrm{T}} z=0, & \text { for all } z \in \operatorname{ker}(M(q)), \\ F(q, \dot{q}, t)^{\mathrm{T}} e \neq 0 & \text { for all } e \in \operatorname{ker}(M(q)), e \neq 0 .\end{cases}
$$

Then the MLCP in (16) has at most one solution $\ddot{q}(t)$. Consequently, if solvability holds, $\nabla h(q) \lambda(t)$ is unique.

Proof The inclusions in (32) are a direct consequence of (c) in Proposition 2. Our first assumption in (b) implies that for all $x \in \Phi, z \in \operatorname{ker}(M(q))$ :

$$
\nabla h(q)^{\mathrm{T}}(x+z)+H(q, \dot{q})=\nabla h(q)^{\mathrm{T}} x+H(q, \dot{q}) \in K,
$$

see (19). One has $\varphi(x+z)=\Psi_{\Phi}(x+z)$ and $\varphi(x)=\Psi_{\Phi}(x)=0$. Thus,

$$
\varphi(x+z)=\Psi_{\Phi}(x+z)=\varphi(x) .
$$

It results that the first condition in (d) of Proposition 2 is satisfied. The second condition in (d) of Proposition 2 is a direct consequence of our second assumption in (b). The uniqueness result comes thus directly from Proposition 2.

The first assumption in (b) is equivalent to

$$
(\forall i \in\{1, \ldots, m\}): \quad \nabla h_{i}(q) \in \operatorname{ker}(M(q))^{\perp} .
$$


Moreover, the matrix $M(q)$ is symmetric and thus $\operatorname{ker}(M(q))^{\perp}=\operatorname{Im}(M(q))$. It results that the first assumption in (b) is equivalent to

$$
(\forall i \in\{1, \ldots, m\}): \quad \nabla h_{i}(q) \in \operatorname{Im}(M(q)) .
$$

The second condition in (32) is equivalent to the uniqueness of $\nabla h(q) \lambda$, the first one to the uniqueness of $M(q) \ddot{q}(t)$, and the third one to the uniqueness of $F(q, \dot{q}, t)^{\mathrm{T}} \ddot{q}(t)$.

Remark 4 The first condition of Proposition 5(b) is equivalently rewritten as $\operatorname{ker}(M(q)) \subseteq$ $\operatorname{ker}\left(\nabla h(q)^{\mathrm{T}}\right) \Leftrightarrow \operatorname{Im}(M(q)) \supseteq \operatorname{Im}(\nabla h(q))$. Obviously, if $M(q)$ has rank $n$, this is always satisfied. The condition requires that the rank of $\nabla h(q)$ be less or equal to that of $M(q)$. In a system with two independent constraints like that of Fig. 3, the uniqueness condition of Proposition 5 thus implies that $\operatorname{rank}(M(q)) \geq 2$. If the system has more than 2 degrees of freedom, it is then allowed that $M(q)$ be singular. Let us go a step further. The above condition is equivalent to $z \notin \operatorname{ker}\left(\nabla h(q)^{\mathrm{T}}\right) \Rightarrow z \notin \operatorname{ker}(M(q))$. Moreau's viability Lemma (see [14, Propositions 5.1 and 5.2], [32]) states that the right velocity $\dot{q}\left(t^{+}\right.$), which is in our case simply equal to $\dot{q}(t)$ since we do not consider impacts, has to satisfy $\dot{q}(t) \in T_{S}(q(t))$ for $q(t) \in S$ to be satisfied (such velocities may be named viable). From (29) it follows that $\nabla h(q)^{\mathrm{T}} \dot{q}(t) \geq 0$. Thus we deduce that $\dot{q}(t) \notin \operatorname{ker}\left(\nabla h(q(t))^{\mathrm{T}}\right) \Leftrightarrow \nabla h(q(t))^{\mathrm{T}} \dot{q}(t)>0 \Rightarrow$ $\dot{q}(t) \notin \operatorname{ker}(M(q(t)))$ : velocities that point strictly inside the admissible set $S$ and which satisfy the condition for uniqueness of accelerations have to produce positive kinetic energy. The solvability condition of Proposition 4(i) yields close conclusions. Indeed, it states that the nonzero viable velocities $\dot{q}(t)$ (i.e. the velocities that belong to the tangent cone) are not in $\operatorname{ker}(M(q))$, consequently they produce positive kinetic energy. These are observations similar to those made in [21] for bilateral constraints (end of Sect. 2 in that paper). The other conditions of Propositions 4 and 5 impose some conditions on the generalized forces for the viable velocities which produce zero kinetic energy.

\section{Mixed bilateral/unilateral frictionless constraints}

It is often the case in practice that the system under study is subjected not only to $m$ unilateral, but also to $p$ bilateral constraints. For the sake of clarity of the presentation, let us denote the set of bilateral holonomic constraints as $f(q)=0$, with $f: \mathbb{R}^{n} \rightarrow \mathbb{R}^{p}$ a differentiable function. The Lagrange multipliers associated with the bilateral constraints are denoted as $\mu \in \mathbb{R}^{p}$. Therefore, the right-hand side of the dynamics in (1) becomes equal to $\nabla h(q) \lambda+\nabla f(q) \mu$ and the MLCP in (16) becomes

$$
\left\{\begin{array}{l}
M(q) x+F(q, \dot{q}, t)-\nabla h(q) \lambda-\nabla f(q) \mu=0, \\
0 \leq \lambda \perp \nabla h(q)^{\mathrm{T}} x+H(q, \dot{q}) \geq 0, \\
\nabla f(q)^{\mathrm{T}} x+\frac{d}{d t}\left(\nabla f(q)^{\mathrm{T}}\right) \dot{q}=0
\end{array}\right.
$$

with $x=\ddot{q}(t)$. It has been shown above that $\nabla h(q) \lambda \in-\partial \Psi_{\Phi}(\ddot{q})$ with $\Phi$ in (19). Quite similarly one may prove that $\nabla f(q) \mu \in-\partial \Psi_{\Omega}(\ddot{q}),{ }^{2}$ with $\Omega=\left\{x \in \mathbb{R}^{n}: \nabla f(q)^{\mathrm{T}} x+\right.$ $\left.\frac{d}{d t}\left(\nabla f(q)^{\mathrm{T}}\right) \dot{q}=0\right\}$. Then, $\nabla h(q) \lambda+\nabla f(q) \mu \in-\partial \Psi_{\Phi}(\ddot{q})-\partial \Psi_{\Omega}(\ddot{q})=-\partial \Psi_{\Phi \cap \Omega}(\ddot{q})$, where the last equality is obtained under the condition that $\Phi \cap \Omega \neq \emptyset$ (which is an equivalent of Assumption 1), see [35, Theorem 23.8]. Proceeding along the same steps as in

\footnotetext{
${ }^{2}$ The minus sign is added here just to be coherent with the unilateral case.
} 
Sect. 3, and replacing $\Phi$ by $\Phi \cap \Omega$, one notes in particular that $(\operatorname{dom}(\varphi))_{\infty}=(\Phi \cap \Omega)_{\infty}=$ $\operatorname{ker}\left(\nabla f(q)^{\mathrm{T}}\right) \cap T_{S}(q)$, where we assumed as above that the $m$ unilateral constraints are active and satisfy the MFCQ. We therefore obtain an extension of Proposition 4:

Proposition 6 (MLCP solvability) Suppose that all $m$ unilateral constraints are active, i.e. $h(q)=0$, that the MFCQ holds, and that the system is subjected to $p$ bilateral holonomic constraints $f(q)=0$. Let Assumption 1 hold, $\Phi \cap \Omega \neq \emptyset$, and that one of the following conditions is satisfied:

(i) $T_{S}(q) \cap \operatorname{ker}\left(\nabla f(q)^{\mathrm{T}}\right) \cap \operatorname{ker}(M(q))=\{0\}$,

(ii) $T_{S}(q) \cap \operatorname{ker}\left(\nabla f(q)^{\mathrm{T}}\right) \cap \operatorname{ker}(M(q)) \neq\{0\}$ and $F(q, \dot{q}, t)^{\mathrm{T}} v>0$ for all $v \in T_{S}(q) \cap$ $\operatorname{ker}(M(q)) \cap \operatorname{ker}\left(\nabla f(q)^{\mathrm{T}}\right), v \neq 0$.

Then the MLCP in (34) has at least one solution.

Another way to treat this extension is to rewrite the bilateral constraints as a set of unilateral constraints: $f(q) \geq 0$ and $-f(q) \geq 0$. This yields the same result. Uniqueness of solutions of the MLCP also follows as an extension of Proposition 5, where one rewrites the second line in (32) as $\lambda_{1}(t)-\lambda_{2}(t) \in \operatorname{ker}(\nabla h(q)), \mu_{1}(t)-\mu_{2}(t) \in \operatorname{ker}(\nabla f(q))$, and the first line in (33) as $\nabla h(q)^{\mathrm{T}} z=0$ and $\nabla f(q)^{\mathrm{T}} z=0$, for all $z \in \operatorname{ker}(M(q))$. This gives conditions such that the MLCP in (34) has at most one solution $x$. Consequently, if solvability holds, $\nabla h(q) \lambda(t)+\nabla f(q) \mu(t)$ is unique.

Remark 5 The contact problem with both bilateral holonomic and unilateral constraints is analyzed in [15], with full rank $M(q)$ and $\nabla f(q)$. Thus the bilateral multiplier $\mu$ can be calculated as a function of $q, \dot{q}$ and $\lambda$ in order to obtain a modified contact LCP.

\section{Transformation of the MLCP into an LCP}

It is also of interest to investigate how the MLCP may be solved. A path is to transform it into an LCP, for which numerical solvers exist. Since $M(q)$ is symmetric positive semidefinite of rank $r$, there exists a unitary matrix $U(q) \in \mathbb{R}^{n \times n}, U(q) U(q)^{\mathrm{T}}=I$, such that $U(q) M(q) U(q)^{\mathrm{T}}=\left(\begin{array}{rr}M_{r}(q) & 0 \\ 0 & 0\end{array}\right)$, where $M_{r}(q)$ is symmetric positive definite (it is even diagonal) [10, Corollary 5.4.4]. After the variable change $y=U(q) x$ one may rewrite the MLCP (16) as

$$
\left\{\begin{array}{l}
M_{r}(q) y_{r}+(U(q) F(q, \dot{q}, t))_{r}=(U(q) \nabla h(q))_{r} \lambda, \\
(U(q) F(q, \dot{q}, t))_{n-r}=(U(q) \nabla h(q))_{n-r} \lambda, \\
0 \leq \lambda \perp(U(q) \nabla h(q))_{r}^{\mathrm{T}} M_{r}(q)^{-1}(U(q) \nabla h(q))_{r} \lambda \\
\quad-(U(q) \nabla h(q))_{r}^{\mathrm{T}} M_{r}(q)^{-1}(U(q) F(q, \dot{q}, t))_{r} \\
\quad+(U(q) \nabla h(q))_{n-r}^{\mathrm{T}} y_{n-r}+H(q, \dot{q}) \geq 0 .
\end{array}\right.
$$

The LCP matrix is the $m \times m$ matrix $(U(q) \nabla h(q))_{r}^{\mathrm{T}} M_{r}(q)^{-1}(U(q) \nabla h(q))_{r}$. For a matrix $A \in \mathbb{R}^{n \times m}$, the submatrix $(A)_{r} \in \mathbb{R}^{r \times m}$ consists of its first $r$ rows. A vector $y \in \mathbb{R}^{n}$ is also decomposed as $y=\left(\begin{array}{c}y_{r} \\ y_{n-r}\end{array}\right)$. If $M(q)$ has full rank $r=n$ then (35) becomes an LCP. This transformation may also be used to obtain the inclusion (similar to (21)):

$$
\left(\begin{array}{c}
M_{r}(q) y_{r}+(U(q) F(q, \dot{q}, t))_{r} \\
(U(q) F(q, \dot{q}, t))_{n-r}
\end{array}\right) \in-\partial \Psi_{\bar{\Phi}}(y),
$$


where $\bar{\Phi}=\left\{y \in \mathbb{R}^{n}: U(q)^{\mathrm{T}} y \in \Phi\right\}=U(q)^{-T}(\Phi)$, and the chain rule of convex analysis (see Theorem 1 in Appendix B) has been used to obtain (36). This transformation does not simplify the expressions of the normal and tangent cones; however, it simplifies the expression of $\operatorname{ker}(M(q))$. As we shall see in Sect. 6, the singularity of $M(q)$ implies that $y_{n-r}=0$ in (35).

\section{The constrained Hamiltonian dynamics}

It is of interest to investigate the Hamiltonian dynamics of systems with a singular mass matrix, in particular the transformation from Lagrangian to Hamiltonian dynamics, and vice versa. The theory of singular Lagrangian and Hamiltonian systems has a long history in Physics, where geometrical, coordinate-free analysis are performed [9, 18, 26, 33]. In particular, equivalence is shown in [9]. We adopt here a different path with generalized (local) coordinates $q$ and quasi-Lagrange dynamics, where basic convex analysis allows us to extend the Legendre transformation to the singular case. It is commonly admitted that Lagrangian and Hamiltonian formalisms are equivalent, and the Fenchel (or Legendre-Fenchel) transformation (5) applied to the system's Lagrangian function allows one to pass from one formalism to the other, under the condition of strict convexity of the Lagrangian function with respect to $\dot{q}[6, \S 15 . \mathrm{A}][36, \S 2.14,2.15]$. When $M(q)$ has full rank $n$, the Legendre-Fenchel transform (5) of the Lagrangian function $L(q, \dot{q}, \lambda)$, defines the Hamiltonian function $H(q, p, \lambda)$, which is the total mechanical energy of the system. One may write $L(q, \dot{q}, \lambda)=$ $\frac{1}{2} \dot{q}^{\mathrm{T}} M(q) \dot{q}-V(q)+h(q)^{\mathrm{T}} \lambda$ and $H(q, p, \lambda)=\frac{1}{2} p^{\mathrm{T}} M(q)^{-1} p+V(q)-h(q)^{\mathrm{T}} \lambda$ where $V(q)$ is the potential energy.

Under the full rank condition $r=n$, one has $L^{\star}(q, \dot{q}, \lambda)=H(q, p, \lambda)$ and by the Fenchel-Moreau theorem [13, Theorem I.10] $L(q, \dot{q}, \lambda)=H^{\star}(q, p, \lambda)$, where both conjugacy calculations are made with $\dot{q}$ and $p$ as variables, respectively. The complementarity Hamiltonian system is given by

$$
\left\{\begin{array}{l}
\dot{q}=\frac{\partial H}{\partial p}=M(q)^{-1} p, \\
\dot{p}=-\frac{\partial H}{\partial q}=-\frac{\partial}{\partial q}\left(\frac{1}{2} p^{\mathrm{T}} M(q)^{-1} p+V(q)\right)+\nabla h(q) \lambda, \\
0 \leq \lambda \perp h(q) \geq 0 .
\end{array}\right.
$$

When $r<n$, the strict convexity is lost, so that the Lagrangian function is a convex degenerate quadratic function of $\dot{q}$. Its Legendre-Fenchel transform $L^{\star}(q, \dot{q}, \lambda)$ may be calculated following the material in [24, Chap. E, Example 1.1.4]. One obtains

$$
\left\{\begin{array}{l}
H(q, p, \lambda)=\frac{1}{2} p^{\mathrm{T}} M(q)^{\dagger} p+V(q)-h(q)^{\mathrm{T}} \lambda, \\
p \in \operatorname{Im}(M(q)),
\end{array}\right.
$$

where $M(q)^{\dagger}$ is the Moore-Penrose generalized inverse of $M(q)$. Since $M(q)^{\dagger}$ is symmetric positive semi-definite, the Hamiltonian function $H(q, p, \lambda)$ is convex in $p$, so its conjugate function is the original Lagrangian function, under the constraint $\dot{q} \in \operatorname{Im}\left(M(q)^{\dagger}\right)$. A constraint appears in (38), reflecting the loss of rank of $M(q)$ that makes the Lagrangian function degenerate, i.e. $\frac{\partial^{2}}{\partial \dot{q}^{2}} L(q, \dot{q}, \lambda)$ is not of full rank. Consequently, the Lagrange dynamics are an implicit system, see Remark 6. If this constraint is not satisfied then $H(q, p, \lambda)=+\infty$. The basic idea of what comes next is to use the diagonalization of 
$M(q)$ as in Sect. 5, so that the kinetic energy (more exactly the kinetic co-energy) expressed in the new variable (a quasi-velocity) has a simple form. To this end, let us introduce the quasi-velocity $v=U(q) \dot{q}=\left(\begin{array}{c}v_{r} \\ v_{n-r}\end{array}\right)$. Then the kinetic energy becomes

$$
\begin{aligned}
T(q, \dot{q})=\frac{1}{2} \dot{q}^{\mathrm{T}} M(q) \dot{q} & =\frac{1}{2} \dot{q}^{\mathrm{T}} U(q)^{\mathrm{T}} U(q) M(q) U(q)^{\mathrm{T}} U(q) \dot{q} \\
& =\frac{1}{2} \dot{q}^{\mathrm{T}} U(q)^{\mathrm{T}}\left(\begin{array}{cc}
M_{r}(q) & 0 \\
0 & 0
\end{array}\right) U(q) \dot{q} \\
& =\frac{1}{2} v_{r}^{\mathrm{T}} M_{r}(q) v_{r}=T\left(q, v_{r}\right)
\end{aligned}
$$

One has $M(q)^{\dagger}=U(q)^{\mathrm{T}}\left(\begin{array}{cc}M_{r}(q)^{-1} & 0 \\ 0 & 0\end{array}\right) U(q)$ [10, Fact 8.15.1 and $\left.\S 6.2\right]$. Thus the kinetic energy is given by

$$
\begin{aligned}
T(q, p)=\frac{1}{2} p^{\mathrm{T}} M(q)^{\dagger} p & =\frac{1}{2} p^{\mathrm{T}} U(q)^{\mathrm{T}} U(q) M(q)^{\dagger} U(q)^{\mathrm{T}} U p \\
& =\frac{1}{2} p^{\mathrm{T}} U(q)^{\mathrm{T}}\left(\begin{array}{cc}
M_{r}(q)^{-1} & 0 \\
0 & 0
\end{array}\right) U(q) p .
\end{aligned}
$$

Let $m=U(q) p$. Doing the same partition as for the quasi velocity, one obtains

$$
T(q, p)=\frac{1}{2} m^{\mathrm{T}}\left(\begin{array}{cc}
M_{r}(q)^{-1} & 0 \\
0 & 0
\end{array}\right) m=\frac{1}{2} m_{r}^{\mathrm{T}} M_{r}(q)^{-1} m_{r} .
$$

Equaling the kinetic energy in (41) and co-energy in (39), one finds that $m_{r}=M_{r}(q) v_{r}$, which extends the relation between the generalized momentum and the generalized velocity for full rank $M(q)$ [6, p. 65] to quasi momentum $m$ and quasi-velocity $v$. If $U(q)$ is a Jacobian then $v$ is a generalized velocity and not merely a quasi-velocity. However, usually this is not the case.

The quasi-Lagrange dynamics is obtained as follows, starting from (1):

$$
\left\{\begin{array}{l}
M_{r}(q) \dot{v}_{r}-M_{r}(q)\left(\dot{U}(q) U(q)^{\mathrm{T}} v\right)_{r}+\left(U(q) F\left(q, U(q)^{\mathrm{T}} v, t\right)\right)_{r}=(U(q) \nabla h(q))_{r} \lambda, \\
\left(U(q) F\left(q, U(q)^{\mathrm{T}} v, t\right)\right)_{n-r}=(U(q) \nabla h(q))_{n-r} \lambda .
\end{array}\right.
$$

It is clear that the loss of rank of $M(q)$ imposes a constraint in the system, represented by the last $n-r$ lines in (42). In the unconstrained case, the nonlinear forces have to satisfy $\left(U(q) F\left(q, U(q)^{\mathrm{T}} v, t\right)\right)_{n-r}=0$. In the unilaterally constrained case, these forces have to comply with (35), where the multiplier $\lambda$ is a solution of the LCP, and $(U(q) F(q, \dot{q}, t))_{n-r}=$ $(U(q) \nabla h(q))_{n-r} \lambda$ with $\lambda \geq 0$.

Remark 6 The structure of the quasi-Lagrange dynamics in (42) differs from other canonical dynamics obtained when the system has full rank inertia matrix, and is subject to holonomic equality constraints. Transformations applied to systems with holonomic constraints usually aim at decoupling the dynamics into a "tangent" (independent of the contact force) and "normal" (contact force dependent) dynamics, using various annihilators of $\nabla h(q)[15,16,27,29]$. Systems with a singular mass matrix are descriptor variable systems, or implicit systems in the quasi-linear form $G(\dot{x}, x, t, \lambda)=E(x) \dot{x}+H(x, t, \lambda)$, where 
$E(x)=\left(\begin{array}{cc}I & 0 \\ 0 & M\left(x_{1}\right)\end{array}\right), x_{1}=q, x_{2}=\dot{q}, x=\left(\begin{array}{l}x_{1} \\ x_{2}\end{array}\right)$, and with $\frac{\partial G}{\partial \dot{x}}(x)=E(x)$ a singular Jacobian matrix (here $E(x)$ has rank $2 n-r)$. Transformations aim at decoupling the dynamics according to the structure of $E(x)$ [44].

Let us now obtain the quasi-Hamilton dynamics. From the fact that $\dot{q}=\frac{\partial H}{\partial p}=\frac{\partial p}{\partial m} \frac{\partial H}{\partial m}$ and $p=U(q)^{\mathrm{T}} m$, one obtains

$$
\left\{\begin{array}{l}
\dot{q}=U(q)^{\mathrm{T}} \frac{\partial H}{\partial m}, \\
\dot{m}=-U(q) \frac{\partial H}{\partial q}-U(q) \dot{U}(q) m, \\
m \in U(q) \operatorname{Im}(M(q))=\operatorname{Im}(U(q) M(q))=\operatorname{Im}\left(U(q) M(q) U(q)^{\mathrm{T}}\right) \Leftrightarrow m=\left(\begin{array}{c}
m_{r} \\
0_{n-r}
\end{array}\right) .
\end{array}\right.
$$

Notice that if $r=n$ then $U(q)=I$, and one recovers (37). The system in (43) is called quasi-Hamiltonian because of the bias $-U(q) \dot{U}(q) m$. The first equation in (43) rewrites $v=\frac{\partial H}{\partial m}$, equivalently $v_{r}=M_{r}(q)^{-1} m_{r}, v_{n-r}=0$. This reveals that $r<n$ imposes the constraint $v_{n-r}=0$ in addition to $m_{n-r}=0$. One infers that $\dot{q} \in U(q)^{\mathrm{T}} \operatorname{Im}\left(U(q) M(q) U(q)^{\mathrm{T}}\right)=$ $\operatorname{Im}(M(q))=\operatorname{Im}\left(M(q)^{\dagger}\right)$, and $p \in U(q)^{\mathrm{T}} \operatorname{Im}\left(U(q) M(q) U(q)^{\mathrm{T}}\right)=\operatorname{Im}(M(q))=\operatorname{Im}\left(M(q)^{\dagger}\right)$ (as known from (38), and we used [10, Proposition 6.1.6(vii)]). Therefore, the admissible generalized velocities and momenta are those which correspond to motions that create kinetic co-energy and energy, respectively. Taking into account the velocity constraint allows one to conclude the reverse of (38): $L(q, \dot{q}, \lambda)=H^{\star}(q, p, \lambda)$.

The generalized momentum constraint of (38) is transformed into an explicit quasimomentum constraint in (43). Starting from (42) the dynamics for $m_{r}$ is obtained as

$$
\begin{aligned}
\dot{m}_{r}= & \dot{M}_{r}(q) M_{r}(q)^{-1} m_{r}+M_{r}(q)\left(\dot{U}(q) U(q)^{\mathrm{T}} v\right)-\left(U(q) F\left(q, U(q)^{\mathrm{T}} v, t\right)\right)_{r} \\
& +(U(q) \nabla h(q))_{r} \lambda .
\end{aligned}
$$

The last $n-r$ lines of the $\dot{m}$ dynamics in (43) correspond to the same equality as in the second line of (42), due to the constraint on $m$.

Remark 7 If $r=0$ then $M(q)=0$ and both $\dot{q}$ and $p$ are identically zero, as well as their derivatives $\ddot{q}$ and $\dot{p}$. The system is in an equilibrium state.

Let us recapitulate the developments of this section. The next dynamical equations are equivalent one to another, where $U(q)$ is a unitary matrix such that $U(q) M(q) U(q)^{\mathrm{T}}=$ $\operatorname{diag}\left(M_{r}(q), 0\right), \operatorname{rank}\left(M_{r}(q)\right)=r<n$ :

$$
\begin{aligned}
& \left\{\begin{array}{l}
M(q) \ddot{q}+F(q, \dot{q}, t)=\nabla h(q) \lambda, \\
0 \leq \lambda \perp h(q) \geq 0, \\
\dot{q} \in \operatorname{Im}(M(q)), \\
L(q, \dot{q}, \lambda)=\frac{1}{2} \dot{q}^{\mathrm{T}} M(q) \dot{q}-V(q)+h(q)^{\mathrm{T}} \lambda
\end{array}\right. \\
& \Leftrightarrow \quad\left\{\begin{array}{l}
\dot{q}=\frac{\partial H}{\partial p}, \quad \dot{p}=-\frac{\partial H}{\partial q}, \\
0 \leq \lambda \perp h(q) \geq 0, \\
p \in \operatorname{Im}(M(q)) \\
H(q, p, \lambda)=\frac{1}{2} p^{\mathrm{T}} M(q)^{\dagger} p+V(q)-h(q)^{\mathrm{T}} \lambda
\end{array}\right.
\end{aligned}
$$




$$
\begin{aligned}
& \Leftrightarrow\left\{\begin{array}{l}
m=U(q) p, \quad m_{n-r}=0, \\
\dot{m}_{r}=\dot{M}_{r}(q) M_{r}(q)^{-1} m_{r}+M_{r}(q)\left(\dot{U}(q) U(q)^{\mathrm{T}} v\right)-\left(U(q) F\left(q, U(q)^{\mathrm{T}} v, t\right)\right)_{r} \\
\quad+(U(q) \nabla h(q))_{r} \lambda \\
\left(U(q) F\left(q, U(q)^{\mathrm{T}} v, t\right)\right)_{n-r}=(U(q) \nabla h(q))_{n-r} \lambda, \\
0 \leq \lambda \perp h(q) \geq 0
\end{array}\right. \\
& \Leftrightarrow\left\{\begin{array}{l}
m=U(q) p, \quad m_{n-r}=0, \quad v=U(q) \dot{q}, \quad v_{n-r}=0 \\
H(q, m, \lambda)=\frac{1}{2} m_{r}^{\mathrm{T}} M_{r}(q)^{-1} m_{r}+V(q)-h(q)^{\mathrm{T}} \lambda, \\
v=\frac{\partial H}{\partial m}, \\
\dot{m}=-U(q) \frac{\partial H}{\partial q}-U(q) \dot{U}(q) m, \\
0 \leq \lambda \perp h(q) \geq 0
\end{array}\right. \\
& \Leftrightarrow\left\{\begin{array}{l}
v=U(q) \dot{q}, v_{n-r}=0, \quad L(q, v, \lambda)=\frac{1}{2} v_{r}^{\mathrm{T}} M_{r}(q) v_{r}-V(q)+h(q)^{\mathrm{T}} \lambda \\
M_{r}(q) \dot{v}_{r}-M_{r}(q)\left(\dot{U}(q) U(q)^{\mathrm{T}} v\right)_{r}+\left(U(q) F\left(q, U(q)^{\mathrm{T}} v, t\right)\right)_{r} \\
\quad=(U(q) \nabla h(q))_{r} \lambda, \\
\left(U(q) F\left(q, U(q)^{\mathrm{T}} v, t\right)\right)_{n-r}=(U(q) \nabla h(q))_{n-r} \lambda, \\
0 \leq \lambda \perp h(q) \geq 0 .
\end{array}\right.
\end{aligned}
$$

The LCP in (35) may be obtained as the contact LCP of (48). The constraints $p_{n-r}=0$ are called the primary constraints in the literature [18, 33]. Finally, the classical equality $H(q, p, \lambda)=p^{\mathrm{T}} \dot{q}-L(q, \dot{q}, \lambda)$ remains valid, and equivalently $H(q, m, \lambda)=m^{\mathrm{T}} v-$ $L(q, v, \lambda)$.

Remark 8 There is nothing fundamentally new in the above developments. They are, however, tailored to multibody systems where the usefulness of coordinate-free, purely geometrical arguments as in $[9,18,26,33]$ stemming from Dirac's original results is unclear when time comes to study contact complementarity problems.

\section{Examples}

Many examples of systems with a singular mass matrix are given in [3-5, 25, 38-41], besides the well-known case when Euler angles are used and the mass matrix of a rigid body is obtained from the Olindes-Rodrigues matrix, rendering it singular at certain configurations [14, Eq. (4.3)], [25, §6]. To illustrate the foregoing developments let us present two simple examples.

\subsection{Example 1}

Let us consider the pendulum dynamics, taken from $[1, \S 3.2 .3]$, as depicted in Fig. 5. Let the coordinates of $G$ be $x$ and $y$ in the Galilean frame $\mathcal{R}_{0}=(\mathbf{i}, \mathbf{j})$, and those of $O$ be $x_{o}$ and $y_{o}$. The kinetic energy of the free pendulum is $T(\dot{\theta}, \dot{x}, \dot{y})=\frac{1}{2} m\left(\dot{x}^{2}+\dot{y}^{2}\right)$, as indeed the moment of inertia at $G$ is zero, all the mass being concentrated at $G$. The potential energy is $V(q)=$ 
Fig. 5 A simple pendulum

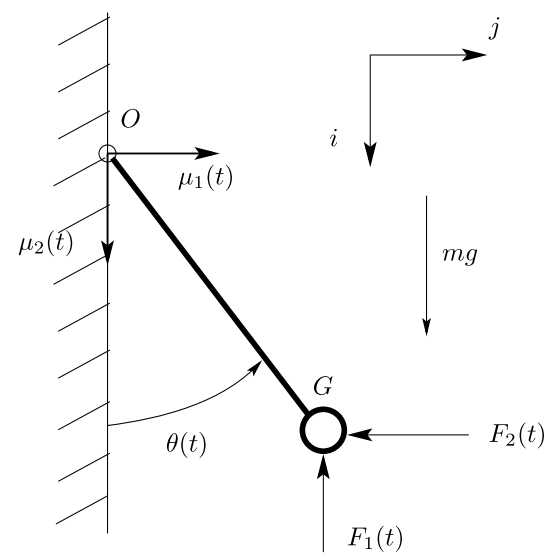

$m g x$. The Lagrangian function is $L(\theta, \dot{\theta}, x, y, \dot{x}, \dot{y})=T(\dot{\theta}, \dot{x}, \dot{y})-V(x)$. Since $O$ is fixed, there are two bilateral constraints $x_{O}=x-l \cos \theta=0, y_{O}=y-l \sin \theta=0$, with which two Lagrange multipliers $\mu_{1}$ and $\mu_{2}$ are respectively associated. Let the unilateral constraint consist of a vertical wall passing through the point $O$, imposing the constraints $h_{1}(q)=$ $y \geq 0, h_{2}(q)=\theta \geq 0$, with which two Lagrange multipliers $\lambda_{1}$ and $\lambda_{2}$ are respectively associated. One has $\lambda=\left(\lambda_{1}, \lambda_{2}\right)^{\mathrm{T}}$ and $\mu=\left(\mu_{1}, \mu_{2}\right)^{\mathrm{T}}$. Thus

$$
\nabla h(q)=\left(\begin{array}{ll}
0 & 0 \\
1 & 0 \\
0 & 1
\end{array}\right)
$$

has rank 2, and the MFCQ holds. Let us choose the generalized coordinates of the free system as $(x, y, \theta)^{\mathrm{T}}$. We can write the Lagrange equations for the pendulum evolving in the plane and subject to the bilateral and unilateral constraints as

$$
\left\{\begin{array}{l}
m \ddot{x}(t)=\mu_{1}(t)+m g+F_{1}(t), \\
m \ddot{y}(t)=\mu_{2}(t)+\lambda_{1}(t)+F_{2}(t), \\
0 . \ddot{\theta}(t)=l \sin \theta \mu_{1}(t)-l \cos \theta \mu_{2}(t)+\lambda_{2}(t), \\
f_{1}(q)=x-l \cos \theta=0, \quad f_{2}(q)=y-l \sin \theta=0, \\
0 \leq \lambda_{1} \perp h_{1}(q)=y \geq 0, \quad 0 \leq \lambda_{2} \perp h_{2}(q)=\theta \geq 0,
\end{array}\right.
$$

where $F=\left(F_{1}, F_{2}\right)^{\mathrm{T}}$ is the force applied at $G$, expressed in $\mathcal{R}_{0}$. Here $M(q)=M=$ $\operatorname{diag}(m, m, 0)$, so

$$
\operatorname{ker}(M)=\left\{z \in \mathbb{R}^{3}: z=\left(\begin{array}{c}
0 \\
0 \\
z_{3}
\end{array}\right), z_{3} \in \mathbb{R}\right\}
$$

and

$$
\operatorname{Im}(M)=\left\{z \in \mathbb{R}^{3}: z=\left(\begin{array}{c}
z_{1} \\
z_{2} \\
0
\end{array}\right), z_{1} \in \mathbb{R}, z_{2} \in \mathbb{R}\right\} .
$$


Fig. 6 Flexible two-degree-of-freedom system (from [40, Fig. 3])
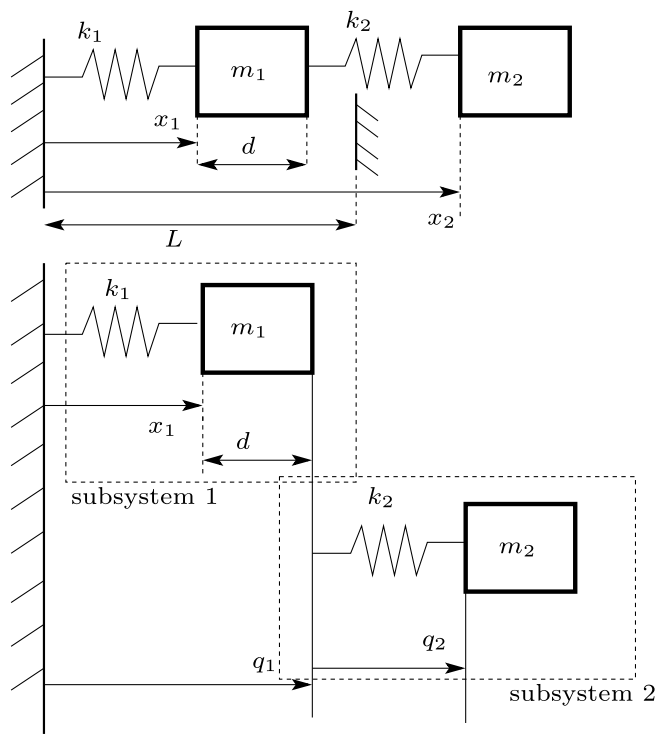

The tangent cone is $T_{S}(q)=\left\{z \in \mathbb{R}^{3}: z_{2} \geq 0, z_{3} \geq 0\right\}$. Finally,

$$
\begin{aligned}
\operatorname{ker}\left(\nabla f(q)^{\mathrm{T}}\right) & =\left\{z \in \mathbb{R}^{3}: z_{1}+z_{3} l \sin \theta=z_{2}-z_{3} l \cos \theta=0\right\} \\
& =\left\{z \in \mathbb{R}^{3}: z=\left(\begin{array}{c}
-l \sin \theta \\
l \cos \theta \\
1
\end{array}\right) z_{3}, z_{3} \in \mathbb{R}\right\} .
\end{aligned}
$$

One obtains $T_{S}(q) \cap \operatorname{ker}(M) \cap \operatorname{ker}\left(\nabla f(q)^{\mathrm{T}}\right)=\{0\}$, hence Proposition 6(i) applies for the MLCP in (34) which is solvable. The uniqueness of $\lambda$ follows from $\operatorname{ker}(\nabla h(q))=\{0\}$, and the uniqueness of $\mu$ follows from $\operatorname{ker}(\nabla f(q))=\{0\}$.

One may also consider the unilateral constraint $h_{3}(q)=-\theta+\pi \geq 0$. However, this does not change the analysis because the unilateral constraints 2 and 3 cannot be active at the same time. Thus the MFCQ still holds despite $\nabla h_{2}(q)$ and $\nabla h_{3}(q)$ are colinear.

\subsection{Example 2}

Let us now consider the system in Fig. 6, taken from [40, 55 (c)]. It consists of two masses $m_{1}>0$ and $m_{2}>0$ connected by linear springs. The unstretched lengths of the springs are $l_{10}$ and $l_{20}$, and their stiffnesses are $k_{1}>0$ and $k_{2}>0$, respectively. According to the notations shown on the figure, one chooses $q=\left(\bar{x}_{1}, q_{1}, \bar{q}_{2}\right)^{\mathrm{T}}$, with $\bar{x}_{1}=x_{1}-l_{10}, \bar{q}_{2}=q_{2}-l_{20}$. One analyzes the system as the interconnection of two subsystems, which are interconnected with a bilateral constraint $f(q)=0$. The unilateral constraint is imposed on mass $m_{1}$ by a rigid barrier at a distance $L$ from the wall. Then one obtains the constrained dynamics:

$$
\left\{\begin{array}{l}
\left(\begin{array}{ccc}
m_{1} & 0 & 0 \\
0 & m_{2} & m_{2} \\
0 & m_{2} & m_{2}
\end{array}\right) \ddot{q}(t)=\left(\begin{array}{c}
-k_{1} \bar{x}_{1}(t) \\
0 \\
-k_{2} \bar{q}_{2}(t)
\end{array}\right)+\left(\begin{array}{c}
-\mu(t)-\lambda(t) \\
\mu(t) \\
0
\end{array}\right), \\
f(q)=q_{1}-\bar{x}_{1}-l_{10}-d=0, \\
0 \leq \lambda \perp h(q)=L-\bar{x}_{1}-l_{10}-d \geq 0 .
\end{array}\right.
$$


One has $\operatorname{ker}\left(\nabla f(q)^{\mathrm{T}}\right)=\left\{z \in \mathbb{R}^{3}: z_{1}=z_{2}\right\}, T_{S}(q)=\left\{z \in \mathbb{R}^{3}:-z_{1} \geq 0\right\}, \operatorname{ker}(M)=\left\{z \in \mathbb{R}^{3}:\right.$ $\left.z_{1}=0, z_{2}=-z_{3}\right\}$ and obtains $T_{S}(q) \cap \operatorname{ker}(M) \cap \operatorname{ker}\left(\nabla f(q)^{\mathrm{T}}\right)=\{0\}$, hence Proposition 6(i) applies for the solvability of the MLCP in (34). The uniqueness of $\lambda$ and $\mu$ follows from the fact that $\operatorname{ker}(\nabla h(q))=\{0\}$ and $\operatorname{ker}(\nabla f(q))=\{0\}$.

Remark 9 It is noteworthy that both examples could be analyzed with a set of independent generalized coordinates, by eliminating the redundant coordinates due to the presence of the bilateral constraints. The singularity of the mass matrix comes from the generalized coordinates redundancy. In practice one often chooses to work with redundant coordinates, assembling subsystems by adding bilateral constraints. It is also often hard, if not impossible, to eliminate redundant constraints.

\section{Conclusions}

The main objective of this article is to analyze the well-posedness of the complementarity problem which arises in unilaterally constrained systems, when the mass matrix and the constraints Jacobian matrix are not of full rank. This gives rise to a mixed linear complementarity problem. Conditions guaranteeing that the acceleration and the contact force Lagrange multiplier exist and are unique are stated. The case when bilateral, holonomic constraints are also present is also analyzed. Moreover, the relationships between the (singular) Lagrangian and the Hamiltonian formalisms are studied.

\section{Appendix A: The Mangasarian-Fromovitz Constraint Qualifications (MFCQ) $[19$, pp. 17, 252]}

Let $K=\left\{x \in \mathbb{R}^{n}: h(x) \geq 0\right\}$, where $h: \mathbb{R}^{n} \rightarrow \mathbb{R}^{m}$ is continuously differentiable, and $K$ is not necessarily convex. Suppose that there exists a vector $v \in \mathbb{R}^{n}$ such that $\nabla h_{i}(x)^{\mathrm{T}} v>0$ for all $i \in \mathcal{I}(x)=\left\{i: h_{i}(x)=0\right\}$. Then the tangent cone to $K$ at $x$, defined as the dual of the normal cone in (14) is equal to the linearization cone $\left\{z \in \mathbb{R}^{n}: z^{\mathrm{T}} \nabla h_{i}(x) \geq 0\right.$, for all $i \in \mathcal{I}(x)\}$.

\section{Appendix B: The chain rule of convex analysis}

Theorem 1 [35, Theorem 23.9] Let $f(x)=h(A x)$ where $h(\cdot)$ is a proper convex function on $\mathbb{R}^{m}$ and $A$ is a linear transformation from $\mathbb{R}^{n}$ to $\mathbb{R}^{m}$. Then if $h(\cdot)$ is polyhedral and $\operatorname{Im}(A)$ contains a point of $\operatorname{dom}(h)$, one has $\partial f(x)=A^{\mathrm{T}} \partial h(A x)$, for all $x$.

\section{References}

1. Acary, V., Brogliato, B.: Numerical Methods for Nonsmooth Dynamical Systems. Lecture Notes in Applied and Computational Mechanics, vol. 35. Springer, Berlin (2008)

2. Addi, K., Brogliato, B., Goeleven, D.: A qualitative mathematical analysis of a class of linear variational inequalities via semi-complementarity problems. Applications in electronics. Math. Program., Ser. A 126(1), 31-67 (2011)

3. Agrawal, S.K.: Inertia matrix singularity of series-chain spatial manipulators with point masses. J. Dyn. Syst. Meas. Control 115(4), 723-725 (1993). doi:10.1115/1.2899204 
4. Agrawal, S.K.: Inertia matrix singularity of planar series-chain manipulators. In: Proceedings of the IEEE Int. Conference on Robotics and Automation, Sacramento, California, USA, April 1991, pp. 102-107 (1991)

5. Agrawal, S.K.: Series-chain planar manipulators: inertial singularities. J. Mech. Des. 115(4), 941-945 (1993). doi:10.1115/1.2919291

6. Arnold, V.I.: Mathematical Methods of Classical Mechanics. Graduate Texts in Mathematics, vol. 60. Springer, Berlin (1989)

7. Aubin, J.P.: Applied Functional Analysis. Wiley, New York (1979)

8. Balakrishnan, A.V.: Vibrating systems with singular mass-inertia matrices. In: Proceedings of First International Conference on Nonlinear Problems in Aviation and Aerospace, Daytona Beach, Florida, USA, 9-11 May 1996 (1996)

9. Batlle, C., Gomis, J., Pons, J.M., Roman-Roy, N.: Equivalence between the Lagrangian and Hamiltonian formalism for constrained systems. J. Math. Phys. 27(12), 2953-2962 (1986)

10. Bernstein, D.S.: Matrix, Mathematics. Theory, Facts, and Formulas with Application to Linear Systems Theory. Princeton University Press, Princeton (2005)

11. Bertsekas, D.P.: Convex Optimization Theory. Athena Scientific, Belmont (2009)

12. Bhat, S.P., Bernstein, D.S.: Second-order systems with singular mass matrix and an extension of Guyan reduction. SIAM J. Matrix Anal. Appl. 17(3), 649-657 (1996)

13. Brezis, H.: Analyse Fonctionnelle. Théorie et Applications, 4th edn. Masson, Paris (1983)

14. Brogliato, B.: Nonsmooth Mechanics. Models, Dynamics and Control, 2nd edn. Springer, London (1999)

15. Brogliato, B.: Inertial couplings between unilateral and bilateral holonomic constraints in frictionless Lagrangian systems. Multibody Syst. Dyn. 29(3), 289-325 (2013). doi:10.1007/s11044-012-9317-8

16. Brogliato, B.: Kinetic quasi-velocities in unilaterally constrained Lagrangian mechanics with impacts and friction. Multibody Syst. Dyn. 32(2), 175-216 (2014). doi:10.1007/s11044-013-9392-5

17. Brogliato, B., Thibault, L.: Well-posedness results for non-autonomous complementarity systems. J. Convex Anal. 17(3-4), 961-990 (2010)

18. Cariñena, J.F.: Theory of singular Lagrangians. Fortschr. Phys. 38(9), 641-679 (1990)

19. Facchinei, F., Pang, J.S.: Finite-Dimensional Variational Inequalities and Complementarity Problems. Volume I. Springer Series in Operations Research. Springer, New York (2003)

20. Fraczek, J., Wojtyra, M.: On the unique solvability of a direct dynamics problem for mechanisms with redundant constraints and Coulomb friction in joints. Mech. Mach. Theory 46, 312-334 (2011)

21. Garcia de Jalon, J., Gutierrez-Lopez, M.D.: Multibody dynamics with redundant constraints and singular mass matrix: existence, uniqueness, and determination of solutions for accelerations and constraints forces. Multibody Syst. Dyn. 30(3), 311-341 (2013). doi:10.1007/s11044-013-9358-7

22. Garcia de Jalon, J., Callejo, A., Hidalgo, A.F.: Efficient solution of Maggi's equations. J. Comput. Nonlinear Dyn. 7, 021003 (2012)

23. Havelkova, M.: A geometric analysis of dynamical systems with singular Lagrangians. Commun. Math. 19, 169-178 (2011)

24. Hiriart-Urruty, J.B., Lemaréchal, C.: Fundamentals of Convex Analysis. Grundlehren Text Editions. Springer, Berlin (2001)

25. Hurtado, J.E., Sinclair, A.J.: Lagrangian mechanics of overparameterized systems. Nonlinear Dyn. 66, 201-212 (2011)

26. Kamimura, K.: Singular Lagrangian and constrained Hamiltonian systems, generalized canonical formalism. Nuovo Cimento B 68(1), 33-54 (1982)

27. Laulusa, A., Bauchau, O.A.: Review of classical approaches for constraint enforcement in multibody systems. J. Comput. Nonlinear Dyn. 3, 011004 (2008)

28. Lötstedt, P.: Mechanical systems of rigid bodies subject to unilateral constraints. SIAM J. Appl. Math. 42(2), 281-296 (1982)

29. McClamroch, N.H., Wang, D.: Feedback stabilization and tracking of constrained robots. IEEE Trans. Autom. Control 33(5), 419-426 (1988)

30. Moreau, J.J.: Les liaisons unilatérales et le principe de Gauss. C. R. Acad. Sci., Paris, 1er Sem. 256(4), 871-874 (1963)

31. Moreau, J.J.: Quadratic programming in mechanics: dynamics of one-sided constraints. SIAM J. Control 4(1), 153-158 (1966)

32. Moreau, J.J.: Unilateral contact and dry friction in finite freedom dynamics. In: Moreau, J.J., Panagiotopoulos, P.D. (eds.) Nonsmooth Mechanics and Applications. Int. Centre for Mechanical Sciences, Courses and Lectures, vol. 302, pp. 1-82. Springer, Berlin (1988)

33. Pons, J.M.: On Dirac's incomplete analysis of gauge transformations. Stud. Hist. Philos. Mod. Phys. 36, 491-518 (2005) 
34. Rockafellar, R.T., Wets, R.J.B.: Variational Analysis. Grundlehren der Mathematischen Wissenschaften, vol. 317. Springer, Berlin (1998)

35. Rockafellar, R.T.: Convex Analysis. Princeton Landmarks in Mathematics (1970)

36. Scheck, F.: Mechanics. From Newton's Laws to Deterministic Chaos, 2nd edn. Springer, Berlin (1994)

37. Shampine, L.F., Reichelt, M.W., Kierzenka, J.A.: Solving index-I DAEs in Matlab and Simulink. SIAM Rev. 41(3), 538-552 (1999)

38. Schutte, A., Udwadia, F.: New approach to the modeling of complex multibody dynamical systems. J. Appl. Mech. 78, 021018 (2011)

39. Simeon, B.: Computational Flexible Multibody Dynamics. A Differential-Algebraic Approach. Springer, Berlin (2013)

40. Udwadia, F.E., Phohomsiri, P.: Explicit equations of motion for constrained mechanical systems with singular mass matrices and applications to multi-body dynamics. Proc. R. Soc. A, Math. Phys. Eng. Sci. 462, 2097-2117 (2006)

41. Udwadia, F.E., Schutte, A.D.: Equations of motion for general constrained systems in Lagrangian mechanics. Acta Mech. 213(1-2), 111-129 (2010)

42. Wojtyra, M.: Joint reaction in rigid body mechanisms with dependent constraints. Mech. Mach. Theory 44, 2265-2278 (2009)

43. Yen, J.: Constrained equations of motion in multibody dynamics as ODEs on manifolds. SIAM J. Numer. Anal. 30(2), 553-568 (1993)

44. Zimmer, G., Meier, J.: On observing nonlinear descriptor systems. Syst. Control Lett. 32, $43-48$ (1997) 